\title{
Molecular Complete Remission Following Ivosidenib in a Patient With an Acute Undifferentiated Leukemia
}

\author{
Sandipkumar H. Patel, MBBSa; Sumithira Vasu, MBBSa; Ling Guo, MD, PhDb; Olivia Lemaster, APRN-CNPa;
} John C. Byrd, MDa; and Alison Walker, MDa

\section{ABSTRACT}

\begin{abstract}
Acute undifferentiated leukemia (AUL) is a subtype of acute leukemias of ambiguous lineage. There is no standard treatment approach for AUL, although acute lymphoblastic leukemia-like regimens for induction therapy have been used. Additional data suggest that AUL may be better treated as acute myeloid leukemia (AML), given their similarities in genetic, cytogenetic, and gene expression patterns. Somatic mutations of IDH1 are found in $7 \%$ to $14 \%$ of patients with AML; however, the patient in this study was the first patient with IDH1-mutated AUL treated with ivosidenib. In this case, a woman aged 39 years was found to have anemia and thrombocytopenia after presenting to her primary care physician with fatigue, weight loss, and persistent infections. During further workup of the cytopenia, she was diagnosed with $A U L$ and received $7+3$ (daunorubicin, $60 \mathrm{mg} / \mathrm{m}^{2} / \mathrm{d}$ intravenously on days $1-3$, and cytarabine, $100 \mathrm{mg} / \mathrm{m}^{2}$ 24-hour continuous intravenous infusion on days 1-7) due to the presence of the IDH1 mutation. Bone marrow biopsy performed on day 14 of $7+3$ showed persistent disease, and ivosidenib was initiated due to severe HLA alloimmunization (panel-reactive antibody, $100 \%$ ) and significant bleeding complications. The patient achieved a complete morphologic and molecular remission on ivosidenib monotherapy despite critical bleeding complications during induction. Targeted therapy using ivosidenib may represent an encouraging therapeutic option in patients with AUL and IDH1 mutations. Additional evaluation of ivosidenib in this subgroup of patients with AUL is needed.
\end{abstract}

J Natl Compr Canc Netw 2020;18(1):6-10 doi: $10.6004 /$ jnccn.2019.7368

aDivision of Hematology, Department of Internal Medicine, and ${ }^{\text {b Department of }}$ Pathology, The Ohio State University Comprehensive Cancer Center,

Columbus, Ohio.
Most cases of acute leukemia can be assigned to myeloid, B, or T lineage according to morphologic features and immunophenotype. However, $3 \%$ to $5 \%$ of all cases of acute leukemias in adults constitute a heterogeneous subset that cannot be definitively assigned to a certain cell lineage. ${ }^{1}$ These entities have been referred to as acute leukemias of ambiguous lineage (ALALs). Acute undifferentiated leukemia (AUL) has been categorized by $\mathrm{WHO}$ as a rare subtype of $\mathrm{ALAL}^{2}$ that expresses no known lineage-specific markers but instead may express only HLA-DR, CD34, and/or CD38, and may be positive for terminal deoxynucleotidyl transferase $(\mathrm{TdT}) .^{3}$ The overall prognosis of AUL is poor, and because of its low incidence, knowledge about AUL is limited in terms of clinical and biological characteristics. No standard treatment approach exists for patients with AUL.

\section{Case Report}

A woman aged 39 years was transferred to a tertiary care center after a bone marrow biopsy revealed $80 \%$ to $90 \%$ blasts. She had been well until 1 month before admission, when she noted fatigue, a 25-pound weight loss with concurrent otitis media, and sinus infections lasting 4 weeks. She was evaluated by her primary care physician and found to have new-onset anemia and thrombocytopenia. She was referred to a local hospital where a CBC confirmed a WBC count of $7.9 \times 10^{3} / \mathrm{mcL}$, hemoglobin level of $6.7 \mathrm{~g} / \mathrm{dL}$, platelet count of $111 \times 10^{3} / \mathrm{mcL}$, and $45 \%$ circulating blasts. A bone marrow aspiration and biopsy performed at the local hospital revealed $80 \%$ to $90 \%$ blasts. The patient was transferred to our hospital for further evaluation and management of acute leukemia. She denied night sweats, rash, ecchymosis, epistaxis, or menorrhagia. She also denied a personal history of cancer and exposure to chemotherapy, ionizing radiation therapy, or petrochemicals. Her family history was significant for liver cancer (father) and melanoma (brother). The patient had a 30 pack-year history of smoking but did not drink alcohol or use illicit drugs. There was no evidence of lymphadenopathy or organomegaly on physical examination. 
Admission laboratories are shown in Table 1. Bone marrow aspirate slides from the outside hospital showed $82 \%$ blasts, $0 \%$ promyelocytes, $0 \%$ myelocytes, $1 \%$ metamyelocytes, 3\% bands/neutrophils, 6\% lymphocytes, $1 \%$ monocytes, $0 \%$ eosinophils, $0 \%$ basophils, $7 \%$ erythroids, and $0 \%$ plasma cells. Blasts were small to medium in size with a high nucleus/cytoplasm ratio, fine chromatin, and variably prominent nucleoli; no Auer rods were observed (Figure 1). Flow cytometric analysis of the marrow aspirate showed $82 \%$ blasts that were positive for CD45, CD34, CD38, CD117, HLA-DR, and TdT, and negative for myeloperoxidase (MPO), cytoplasmic CD3, cytoplasmic CD79a (9\%), CD19, cytoplasmic CD22, CD13 (7\%), CD20 (7\%), CD25 (8\%), CD33, CD11b, CD11c, CD14, CD10, CD2, CD5, CD7, CD41, CD56, CD64, and glycophorin (Figure 2). Cytogenetic analysis revealed a normal female karyotype. Fluorescence in situ hybridization was negative for $R U N X 1 T 1 / R U N X 1$ (8q22/21q22), KMT2A (11q23), PML/RARA (15q24/17q21), CBFB (16q22), and TP53 (17p13.1).

The patient was diagnosed with AUL based on the WHO classification, which includes leukemic cells that are not positive for any lineage-specific markers (myeloid lineage: MPO [flow cytometry]; B-cell lineage: CD19, CD79a, cytoplasmic CD22 or CD10; T-cell lineage: cytoplasmic CD3 or surface CD3). ${ }^{4}$ A targeted sequencing panel revealed mutations in $I D H 1 R 132 G$ (variant allele frequency [VAF], 0.30), BCOR G1520V (VAF, 0.28), BCOR
G1520C (VAF, 0.27), and a variant of unknown significance of RUNX1 G135D (VAF, 0.53).

The patient was initially treated with $7+3$ (cytarabine, $100 \mathrm{mg} / \mathrm{m}^{2}$ 24-hour continuous intravenous infusion on days $1-7$ and daunorubicin, $60 \mathrm{mg} / \mathrm{m}^{2} / \mathrm{d}$ intravenously on days 1-3) because of the presence of the $I D H 1$ mutation, which is present in $7 \%$ to $14 \%$ of patients with acute myeloid leukemia (AML). ${ }^{5}$ On day 8 of $7+3$ treatment, she developed hemoptysis and blurry vision due to bilateral preretinal hemorrhages. She subsequently experienced various bleeding complications, including right-sided cerebellar hemorrhage, epistaxis, severe oral mucosal bleeding, and conjunctival hemorrhage. Her platelet counts were trending down and then became undetectable during this time (Figure 3). She received intravenous aminocaproic acid and HLA-matched platelets but experienced no improvement in platelet count; she was found to have transfusion-refractory thrombocytopenia due to severe HLA alloimmunization (panel-reactive antibody, 100\%).

Published incidence of platelet transfusion refractoriness in patients with AML is approximately $5 \%$ and is more frequently observed in parous women, increasing the risk of overall deaths from bleeding events. ${ }^{6}$ This patient had 2 children but had never received packed RBCs or platelet transfusions before her leukemia diagnosis. A bone marrow biopsy on day 14 of $7+3$ treatment confirmed persistent AUL in a fibrotic hypocellular bone marrow (25\%), with $15 \%$ blasts (Figure 4). The blasts expressed HLA-DR and CD34, with subpopulations expressing CD19 and CD11b.

\begin{tabular}{|c|c|c|c|c|c|c|c|}
\hline & $\begin{array}{c}\text { Reference Range, } \\
\text { Adults }\end{array}$ & $\begin{array}{c}\text { On } \\
\text { Admission }\end{array}$ & $\begin{array}{c}\text { Day } 7 \text { on } \\
7+3\end{array}$ & $\begin{array}{l}\text { Day } 14 \text { on } \\
\quad 7+3\end{array}$ & $\begin{array}{l}\text { Day } 9 \text { on } \\
\text { Ivosidenib }\end{array}$ & $\begin{array}{l}\text { Day } 17 \text { on } \\
\text { Ivosidenib }\end{array}$ & $\begin{array}{l}\text { Day } 35 \text { on } \\
\text { Ivosidenib }\end{array}$ \\
\hline \multicolumn{8}{|l|}{$\mathrm{CBC}$} \\
\hline $\mathrm{Hb}, \mathrm{g} / \mathrm{dL}$ & $11.4-15.2$ & 8.1 & 7.2 & 6.8 & 5.9 & 8.5 & 12.3 \\
\hline $\mathrm{RBC}, \mathrm{M} / \mathrm{mcL}$ & $3.91-5.04$ & 2.59 & 2.36 & 2.20 & 2.00 & 2.88 & 4.11 \\
\hline $\mathrm{WBC}, \times 10^{3} / \mathrm{mcL}$ & $3.99-11.19$ & 6.96 & 1.26 & 0.59 & 0.54 & 1.13 & 3.95 \\
\hline Platelets, $\times 10^{3} / \mathrm{mcL}$ & $150-393$ & 51 & 13 & $<5$ & $<5$ & 7 & 237 \\
\hline \multicolumn{8}{|l|}{ Differential count, $\times 10^{3} / \mathrm{mcL}$} \\
\hline Neutrophils segs + bands, absolute & $1.64-7.28$ & 0.14 & & & 0.04 & 0.02 & 0.99 \\
\hline Lymphocytes & $1.16-3.51$ & 1.74 & & & 0.41 & 1.10 & 2.53 \\
\hline Monocytes & $0.22-0.87$ & 0.00 & & & 0.04 & 0.00 & 0.16 \\
\hline Eosinophils & $0.00-0.42$ & 0.00 & & & 0.00 & 0.00 & 0.00 \\
\hline Basophils & $0.00-0.15$ & 0.00 & & & 0.00 & 0.00 & 0.00 \\
\hline Blasts & & 5.08 & & & 0.04 & 0.01 & 0.04 \\
\hline \multicolumn{8}{|l|}{ Coagulation panel } \\
\hline PT, s & $11.9-14.2$ & 14.2 & 14.5 & 19.9 & 17.7 & 14.7 & \\
\hline INR & $0.9-1.1$ & 1.1 & 1.1 & 1.7 & 1.5 & 1.1 & \\
\hline PTT, s & $24.0-34.3$ & 39.6 & 36.1 & 65.9 & 41.6 & 30.4 & \\
\hline Fibrinogen, mg/dL & $220-410$ & 613 & & 785 & 454 & 208 & \\
\hline
\end{tabular}

Abbreviations: $\mathrm{Hb}$, hemoglobin; INR, international normalized ratio; PT, prothrombin time; PTT, partial thromboplastin time; segs, segmented neutrophils. 


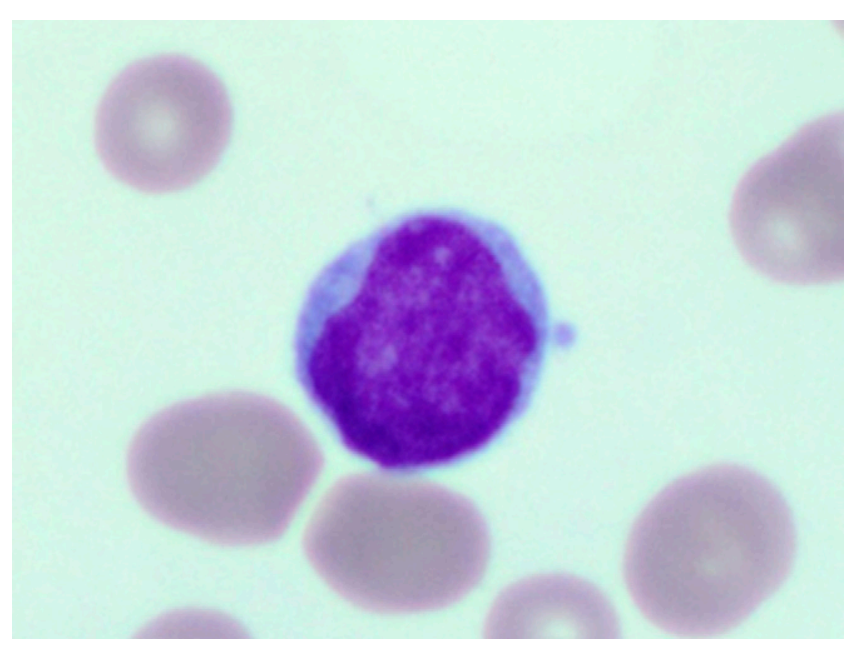

Figure 1. Blast morphology (Wright-Giemsa, original magnification $\times 100$ ).

The targeted sequencing panel after completion of $7+3$ therapy was not obtained because of paucicellular and hemodiluted bone marrow aspirate and a clot section that only showed blood without bone marrow elements.
Because of the patient's persistent disease and the presence of an $I D H 1$ mutation at diagnosis, on day 17 of $7+3$ she was started on ivosidenib, $500 \mathrm{mg} / \mathrm{d}$ rather than a $5+2$ reinduction due to her significant life-threatening bleeding complications and platelet-refractory thrombocytopenia. On day 19 of $7+3$ therapy she declined further, with an increase in the work of breathing, and developed hypoxemia, requiring mechanical ventilator support. Bronchoscopy with bronchoalveolar lavage and cytology were significant for diffuse alveolar hemorrhage, and the patient was treated with methylprednisolone, $1 \mathrm{~g} / \mathrm{d}$ aggressive transfusion support, and a furosemide drip for significant volume overload secondary to multiple blood transfusions. Due to her decline, she was transitioned to DoNot-Resuscitate Comfort Care-Arrest status as per family wishes. The patient received continuous ivosidenib through a nasogastric tube during this period of critical illness and her blood cell counts began to recover (Figure 3, Table 1) after 14 days of ivosidenib; hemorrhagic complications resolved and she recovered without neurologic sequelae.

On discharge from the hospital, the patient had experienced improvement in her vision and was on room air.
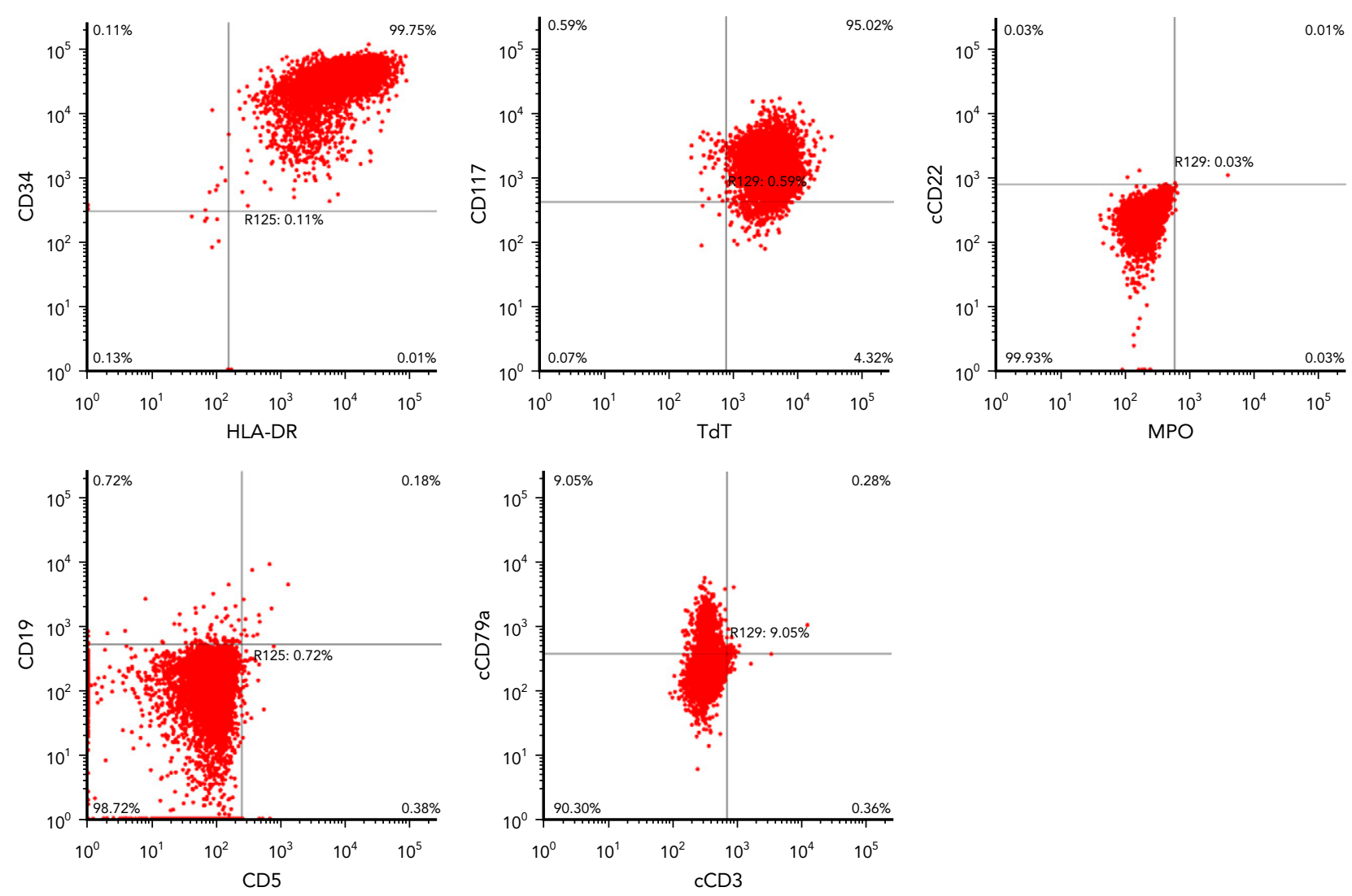

Figure 2. Flow cytometric analysis showed that blasts were positive for (A) CD34 and HLA-DR and (B) CD117 and TdT, and negative for (C) MPO and cCD22, (D) CD5 and CD19, and (E) cCD3 and cCD79a.

Abbreviations: $\mathrm{CCD} 22$, cytoplasmic CD22; cCD3, cytoplasmic CD3; CCD79a, cytoplasmic CD79a; MPO, myeloperoxidase; $R$, residue; TdT, terminal deoxynucleotidyl transferase. 


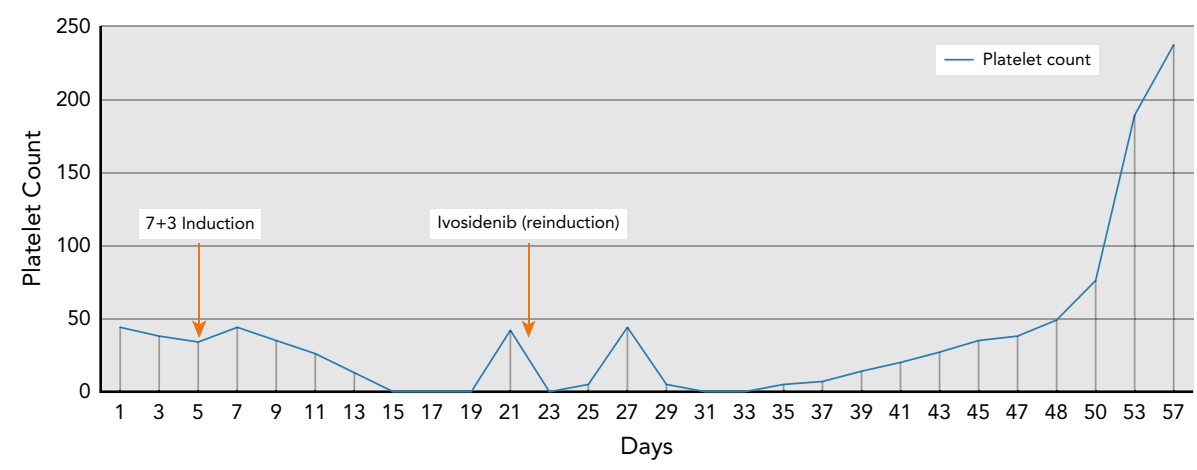

Figure 3. Timeline of platelet counts during treatment course.

Improvement in preretinal hemorrhages in both eyes was also observed during an outpatient ophthalmology examination. On day 31 of ivosidenib, her platelet count was $18910^{3} / \mathrm{mcL}$, and on day 34 , a bone marrow biopsy confirmed a complete remission (CR) with hematologic recovery with $0 \%$ blasts, and the targeted sequencing panel did not detect IDH1 R132G, BCOR G1520V, BCOR G1520C, or RUNX1 G135D mutations.

\section{Discussion}

ALAL is a rare subset of acute leukemia that cannot be classified as having either myeloid or lymphoid lineage despite comprehensive immunophenotyping. ALAL represents a heterogeneous group of rare leukemias, and according to the 2016 update of the WHO classification it is categorized into 5 entities: AUL; mixed-phenotype acute leukemia (MPAL) with $\mathrm{t}(9 ; 22)(\mathrm{q} 34.1 ; \mathrm{q} 11.2)$ (Philadelphia chromosome/BCR-ABL); MPAL with $\mathrm{t}(\mathrm{v} ; 11 \mathrm{q} 23.3)$ (KMT2A/MLL rearrangement); MPAL, B/myeloid, not otherwise specified (NOS); and MPAL, T/myeloid, NOS. ${ }^{1}$ Overall, patients with ALAL have relatively inferior outcomes compared with those with AML or acute lymphoblastic leukemia (ALL). ${ }^{7}$ In one study, patients with AUL were characterized by frequent expression of CD34, TdT, and HLA-DR, supporting the stem cell-like characteristics of this leukemia subtype ${ }^{1}$ and consistent with our patient's presentation. Lao et $\mathrm{al}^{7}$ reported that patients with AUL frequently harbor myelodysplastic syndromesrelated chromosomal alterations, such as del $5 / 5 \mathrm{q}$, del $7 / 7 \mathrm{q}$, and del17/17p, and mutations in DNMT3A, spliceosome (ZRSR2, F236Vfs*6), RUNX1 (frameshift-deletion, T214Pfs*23), cohesin complex (STAG2 and RAD21), and TP53. A high incidence of chromosomal abnormalities, including $\operatorname{del}(20 \mathrm{q})$, tetrasomy 8 and $7,5 q$ deletion, trisomy 13, and trisomy $12 q^{8-10}$ has also been reported in AUL. Our patient was found to have a normal karyotype in both blood and bone marrow aspiration samples.

Standard treatment approaches for patients with AUL have yet to be established. Most published reports have analyzed the treatment of AUL together with that of MPAL despite different clinical characteristics that support an ALL-like regimen for induction therapy for patients with MPAL. ${ }^{1,11,12}$ However, Heesch et $\mathrm{al}^{1}$ reported that $25 \%$ of patients with AUL achieved CR using ALL-like therapy, whereas $40 \%$ achieved CR using AMLlike therapy. We chose to treat our patient using an AML regimen because of the presence of the $I D H 1$ mutation at diagnosis. Point mutations in $I D H 1$ and $I D H 2$ are found in multiple cancer types, including glioma, cholangiocarcinoma, chondrosarcoma, and AML. ${ }^{13}$ IDH2 mutations are more frequent in AML, affecting $8 \%$ to $19 \%$ of patients, compared with $7 \%$ to $14 \%$ for

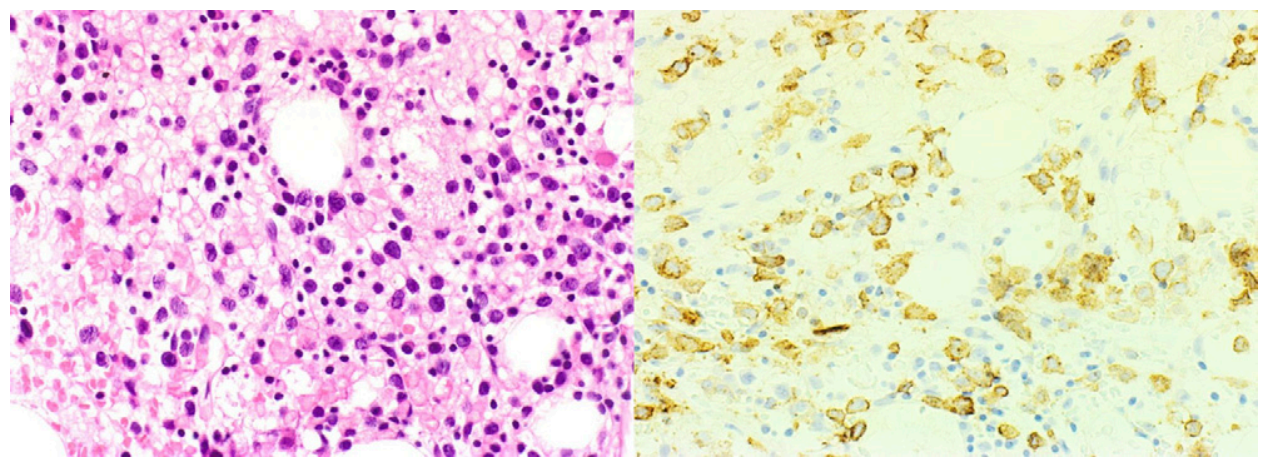

Figure 4. Bone marrow biopsy performed on day 14 of $7+3$ treatment showed that blasts were $(A)$ increased (hematoxylin-eosin, original magnification $\times 40$ ) and $(B)$ positive for CD34 (immunohistochemical, original magnification $\times 40$ ). 
IDH1 mutations. ${ }^{5}$ Mutations in $I D H 1$ induce amino acid changes, most commonly involving a cysteine or histidine substitution for arginine at residue 132 (R132C and $\mathrm{R} 132 \mathrm{H}$, respectively), ${ }^{14}$ resulting in production of 2-hydroxyglutarate $(R-2-\mathrm{HG})$ instead of alpha-ketoglutarate with neomorphic enzymatic activity. This oncometabolite, R-2-HG, modifies DNA and histone methylation and DNA hypermethylation by inhibiting TET2, ${ }^{15}$ resulting in impaired hematopoietic cell differentiation, proliferation, and DNA damage.

IDH1 mutations have been classified as driver mutations and are acquired early in leukemogenesis, suggesting that inhibition of the mutation may more effectively target the leukemic clone. ${ }^{16}$ Ivosidenib is a small molecule inhibitor of mutant IDHI that suppresses 2-HG production, thereby releasing the differentiation block in dysfunctional mutant $I D H$ blast cells and allowing them to continue differentiation into normal functional cells. ${ }^{13}$ DiNardo et al ${ }^{16}$ reported that in patients with advanced IDHI-mutated relapsed or refractory AML, ivosidenib at $500 \mathrm{mg} / \mathrm{d}$ was associated with CR or CR with a partial hematologic recovery rate of $30.4 \%$ (95\% CI, 22.5-39.3), an overall response rate of $41.6 \%$ (95\% CI, 32.9-50.8), and a median CR duration of 9.3 months (95\% CI, 5.6-18.3). On July 20, 2018, the FDA approved ivosidenib for the treatment of patients with relapsed or refractory AML with a susceptible $I D H 1$ mutation, ${ }^{17}$ and more recently on May 2, 2019, for patients with newly diagnosed AML with IDH1 mutation who are aged $\geq 75$ years or have comorbidities that preclude the use of intensive induction chemotherapy, ${ }^{18}$ based on an open-label, single-arm, multicenter clinical trial (AG120C-001; ClinicalTrials.gov identifier: NCT02074839).

For our patient, we chose ivosidenib for reinduction therapy rather than a $5+2$ regimen due to the dominant VAF IDHI mutation and life-threatening bleeding episodes from platelet transfusion-refractory thrombocytopenia. The patient responded exceptionally well to ivosidenib, with recovery of blood counts, including platelets (Figure 3, Table 1), and complete morphologic and molecular responses noted on bone marrow biopsy and the targeted sequencing panel, respectively. Given the morphologic and molecular remission achieved with ivosidenib in this patient, it may be reasonable to consider targeted therapy with ivosidenib for patients with AUL and dominant mutations of IDH1. However, a prospective study is needed to further investigate the role of ivosidenib in patients with AUL and $I D H 1$ mutations.

Submitted July 31, 2019; accepted for publication October 14, 2019

Disclosures: The authors have disclosed that they have not received any financial consideration from any person or organization to support the preparation, analysis, results, or discussion of this article.

Correspondence: Sandipkumar H. Patel, MBBS, Division of Hematology, Department of Internal Medicine, The Ohio State University Comprehensive Cancer Center, A350C Starling-Loving Hall, 320 West 10th Avenue, Columbus, $\mathrm{OH}$ 43210. Email: sandipkumar.patel@osumc.edu

\section{References}

1. Heesch S, Neumann M, Schwartz S, et al. Acute leukemias of ambiguous lineage in adults: molecular and clinical characterization. Ann Hematol 2013;92:747-758.

2. Arber DA, Orazi A, Hasserjian R, et al. The 2016 revision to the World Health Organization classification of myeloid neoplasms and acute leukemia. Blood 2016;127:2391-2405.

3. Béné MC, Porwit A. Acute leukemias of ambiguous lineage. Semin Diagn Pathol 2012;29:12-18.

4. Kurosawa $S$, Toya $T$, Kishida $Y$, et al. Outcome of patients with acute undifferentiated leukemia after allogeneic hematopoietic stem cell transplantation. Leuk Lymphoma 2018;59:3006-3009.

5. Montalban-Bravo G, DiNardo CD. The role of IDH mutations in acute myeloid leukemia. Future Oncol 2018;14:979-993.

6. Comont T, Tavitian S, Bardiaux L, et al. Platelet transfusion refractoriness in patients with acute myeloid leukemia treated by intensive chemotherapy. Leuk Res 2017;61:62-67.

7. Lao ZT, Ding LW, An O, et al. Mutational and transcriptomic profiling of acute leukemia of ambiguous lineage reveals obscure but clinically important lineage bias. Haematologica 2019;104:e200-203.

8. Brito-Babapulle F, Pullon H, Layton DM, et al. Clinicopathological features of acute undifferentiated leukaemia with a stem cell phenotype. $\mathrm{Br} \mathrm{J}$ Haematol 1990;76:210-214

9. Cuneo A, Ferrant A, Michaux JL, et al. Cytogenetic and clinicobiological features of acute leukemia with stem cell phenotype: study of nine cases. Cancer Genet Cytogenet 1996;92:31-36.

10. LeMaistre A, Childs CC, Hirsch-Ginsberg C, et al. Heterogeneity in acute undifferentiated leukemia. Hematol Pathol 1988;2:79-90.
11. Liu QF, Fan ZP, Wu MQ, et al. Allo-HSCT for acute leukemia of ambiguous lineage in adults: the comparison between standard conditioning and intensified conditioning regimens. Ann Hematol 2013;92:679-687.

12. Guru Murthy GS, Dhakal I, Lee JY, et al. Acute leukemia of ambiguous lineage in elderly patients-analysis of survival using Surveillance Epidemiology and End Results-Medicare database. Clin Lymphoma Myeloma Leuk 2017;17:100-107.

13. Dang L, Su SM. Isocitrate dehydrogenase mutation and (R)-2hydroxyglutarate: from basic discovery to therapeutics development. Annu Rev Biochem 2017;86:305-331.

14. Marcucci G, Maharry $K, W u ~ Y Z$, et al. IDH1 and IDH2 gene mutations identify novel molecular subsets within de novo cytogenetically normal acute myeloid leukemia: a Cancer and Leukemia Group B study. J Clin Oncol 2010;28:2348-2355.

15. Guo JU, Su Y, Zhong C, et al. Emerging roles of TET proteins and 5 -hydroxymethylcytosines in active DNA demethylation and beyond. Cell Cycle 2011;10:2662-2668

16. DiNardo CD, Stein EM, de Botton $S$, et al. Durable remissions with ivosidenib in IDH1-mutated relapsed or refractory AML. N Engl J Med 2018;378:2386-2398.

17. Norsworthy KJ, Luo L, Hsu V, et al. FDA approval summary: ivosidenib for relapsed or refractory acute myeloid leukemia with an isocitrate dehydrogenase-1 mutation. Clin Cancer Res 2019;25: 3205-3209.

18. Ivosidenib [package insert]. Cambridge, MA: Agios Pharmaceuticals, Inc; 2019. 